\title{
BAND GAP STABILITY IN KONDO INSULATORS
}

\author{
A. Ślebarski
}

Institute of Physics, University of Silesia, Uniwersytecka 4, 40-007 Katowice, Poland

\section{A. JEZIERSKI}

Institute of Molecular Physics, Polish Academy of Sciences

Smoluchowskiego 17, 60-179 Poznań, Poland

\section{AND A. ZYGMUNT}

Institute for Low Temperature and Structure Research, Polish Academy of Sciences Okólna 2, 50-950 Wrocław, Poland

\begin{abstract}
We report on magnetic measurements and electronic structure investigations of CeNiSn and CeRhSb. Both belong to the group of Kondo insulators. The magnetic susceptibility shows the nonmagnetic ground state for these compounds and their alloys. The $3 d \mathrm{X}$-ray photoemission spectroscopy spectra show evidence for the mixed valence state of Ce in CeRhSb alloys, as also seen for CeNiSn, whereas the spectra for the La substituted (Ce, La) NiSn compounds show only evidence for a pure $\mathrm{Ce}^{3+}$ ground state. We suggest the presence of Kondo-hole states in $(\mathrm{Ce}, \mathrm{La}) \mathrm{RhSb}$. The location of the pseudogap in CeRhSb varies with the number of free electron, the valence of Ce, and the $f-d$ hybridization. We discuss the similar crystallographic properties and the closed electronic structures of ZrNiSn-type semi-Heusler alloys and CeNiSn-type Kondo insulators.
\end{abstract}

PACS numbers: 71.20.-b, 71.20.Eh

\section{Introduction}

Ce-based heavy-fermion metals, which are characterized by large Sommerfeld constants, exhibit various metallic ground states, beginning from Pauli paramagnetic to superconducting. Among the strongly correlated $f$-electron systems are stoichiometric compounds with small-gap semiconductor properties, which might be expected to be metallic, judging from the properties of their $f$-series analogous. The discovery of several Sm-, Tm-, Yb-, and U-based Kondo insulators, e.g., $\mathrm{SmS}$ [1], $\mathrm{SmB}_{6}$ [2], TmSe [3], $\mathrm{YbB}_{12}$ [4], $\mathrm{UFe}_{4} \mathrm{P}_{12}$ [5], UNiSn [6], and FeSi [7], has renewed and enhanced the interest in this subject.

We are concentrated here on the Ce-based intermetallics with a coherent ground state (the gap) in the Kondo lattice. Following extensive experimental 
work on CeNiSn [8], CeRhSb [9], and $\mathrm{Ce}_{3} \mathrm{Bi}_{4} \mathrm{Pd}_{3}$ [10], the term "Kondo insulator" is attributed to an intermetallic compound which: has a nonmagnetic ground state, has an insulating gap due to many-body interactions between conduction and localized electrons in the $f$ shell, and has low-lying excitation which exhibits properties of strong correlation. These properties distinguish Kondo insulators from Mott or charge transfer insulators. We shall discuss it later.

These compounds can be regarded as highly renormalized band insulators, where $f$-electrons behave as valence electrons. Recently, we have discussed the mixed valence of $\mathrm{Ce}$ in $\mathrm{CeNiSn}$ and $\mathrm{CeRhSb}$ [11-13], which seems to be very important for the formation of the gap. In this class of compounds in almost every case there is an isostructural semiconductor in which the $4 f$-element is replaced by a non- $4 f$ tetravalent element.

In terms of a simple Kondo model, the gap would correspond to the filling of the Brillouin zone by additional states due to the Abrikosov-Suhl resonance [14]. In Ref. [15] Fisk et al. argued that the hybridization involves one occupied $f$-state crossing exactly one half-filled conduction band, crossing more than one band leads to a metallic state. At $T<T_{\mathrm{K}}$, where $T_{\mathrm{K}}$ is the Kondo temperature, the Kondo resonance forms a filled Brillouin zone and a gap of size order $k_{\mathrm{B}} T_{\mathrm{K}}$ separates the filled states from the lowest empty one. The $T>T_{\mathrm{K}}$ region was attributed inside this model as a thermally-induced appearence of a localized $f$-electron. Therefore, the materials which support the development of a hybridization gap need to have a simple band structure near the Fermi level as it will be discussed.

An insulating gap formation is indeed theoretically expected for Kondo systems without long-range magnetic order and with a certain Coulomb interaction $U$ between the $f$-states $(U \neq 0)[16]$. However, several experiments give evidence for the ground state with the antiferromagnetic correlations. This problem is also discussed here.

Previous alloying studies show that the energy gap in Ce-based Kondo insulators is very sensitive to the partial substitution of either Ce by La or Ni (Rh) by a transition metal [17-20]. The replacing of the Ce ions by trivalent ions leads to ordinary metals. The replacing of the Ce ions by tetravalent non- $f$ ions such as $\mathrm{Zr}$, Ti or Hf leads to semiconductors. This predicts a ground state of the known Kondo insulators as a result of the hybridization of the one occupied $f$ state with a half-filled conduction band. High-pressure measurements on CeNiSn and CeRhSb showed the strong reduction of the gap due to increasing hybridization with decreasing the volume of the unit cell [21].

In this work we present the electronic structure and magnetic properties of $\mathrm{CeNiSn}$ and CeRhSb. The main goal of this presentation is to find the influence of the valence of Ce-ions $(v>3)$, its partial substitution by trivalent metal (Kondo hole), and the possible magnetic correlations on the coherent gap formation at $\epsilon_{F}$ in the Ce-Kondo insulators. We also discuss the similar crystallographic properties and the closed electronic structures of ZrNiSn-type semi-Heusler alloys and CeNiSn-type Kondo insulators. 


\section{Experimental}

The samples were arc melted on a cooled copper crucible in a high purity argon atmosphere and remelted ten times, and then annealed at $800^{\circ} \mathrm{C}$ for one week. The $\mathrm{X}$-ray analysis showed single phase samples within the usual resolution of $6 \%$. The lattice parameters were investigated as a function of temperature between 8 and $300 \mathrm{~K}$ using the Siemens D-500 diffractometer. A SQUID magnetometer and vibrating magnetometer were used to obtain the magnetization results for low temperatures from $1.6 \mathrm{~K}$ up to $300 \mathrm{~K}$ and magnetic fields of $50 \mathrm{Oe}$ to $50 \mathrm{kOe}$. The ultraviolet photoemission spectra (UPS) and X-ray photoelectron spectra (XPS) were obtained with monochromatized $\mathrm{Al} K_{\alpha}$ radiation at room temperature using a PHI 5700 ESCA spectrometer. The spectra were measured immediately after cleaving the sample in vacuum below $6 \times 10^{-10}$ torr. The electronic structure of the ordered alloys were studied by the self-consistent tight binding linearized muffin-tin orbital method [22] within the atomic sphere approximation (ASA) and the local spin density (LSD) approximation. The exchange correlation potential was assumed in the form proposed by von Barth-Hedin [23] and Langreth-Mehl-Hu (LMH) [24] with corrections included. In the band calculations we assumed the initial configurations according to the periodic table of elements. The values of the atomic sphere radii were taken in such a way that the sum of all atomic sphere volumes was equal to the volume of the unit cell.

\section{Band gap stability in the ternary Ce-intermetallic compounds}

\subsection{Electronic structure of CeNiSn and CeRhSb}

Transport properties of CeNiSn, CeRhSb or $\mathrm{Ce}_{3} \mathrm{Bi}_{4} \mathrm{Pt}_{3}$ show Kondo behavior at high temperatures and formation of a gap at low temperatures. The transport gap is approximately $10 \mathrm{~K}$ and is unstable against any change in $4 f$-conduction electron hybridization caused by alloying [25, 26] and application of pressure $[27,21,28]$ or magnetic field $[18,29]$. In Fig. 1 we present the band structure of CeNiSn plotted along various symmetry directions. The bands cross the Fermi level $\left(\epsilon_{\mathrm{F}}\right)$ in $\Gamma-X$ and $\Gamma-Y$ directions. Therefore, CeNiSn is at $T=0$ more a semimetal with an indirect gap than an insulator. In Fig. 2 we compare the density of states (DOS) with the XPS valence band spectra of CeNiSn. The spectrum is characterized by the major peak located at about 1.5 due to Ni $d$-states. A half width of the VB XPS peak is about twice as wide in CeRhSb than in CeNiSn (Fig. 3), this indicates different partial distributions of the $d$-states in the bands and suggests different intersite hybridization mainly between $\mathrm{Ce} 4 f$ - and $d$-band states. In our recent works we argued that the $f-d$ hybridization determines the formation of the gap at $\epsilon_{\mathrm{F}}$. From the TB LMTO calculations we obtained a pseudo V-shape gap (Figs. 1, 2) located at the Fermi level. Our calculations agree with NMR experiment [30] and always predict the small DOS at $\epsilon_{\mathrm{F}}$. However, a standard resolution of only $0.4 \mathrm{eV}$ of the measured VB XPS spectra makes discussing of the Kondo-insulator behavior impossible. The spectra are detected at room temperature, while the calculations have been done for the ground state. In our experiment we can, however, evaluate the distribution of partial DOS in the XPS valence bands 


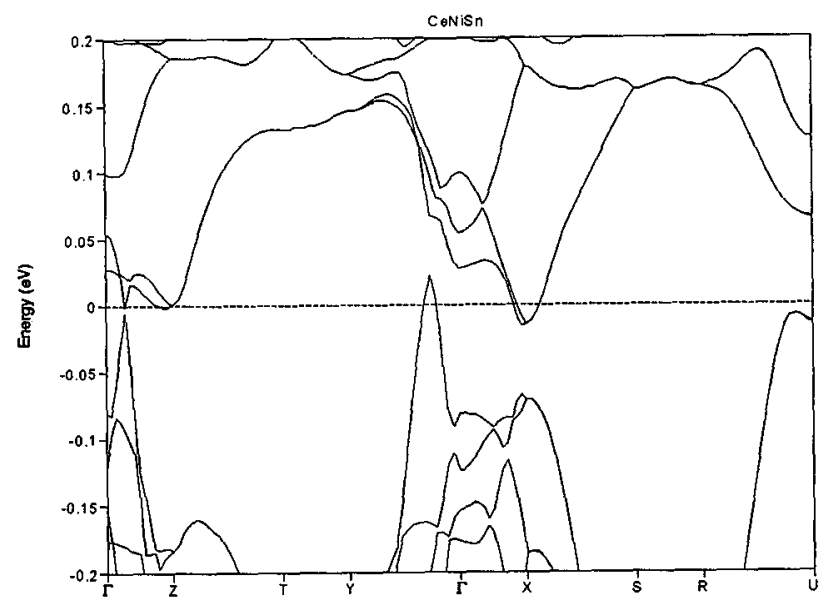

Fig. 1. TB LMTO band structure of CeNiSn along various symmetry directions in the Brillouin zone (from Ref. [19]).

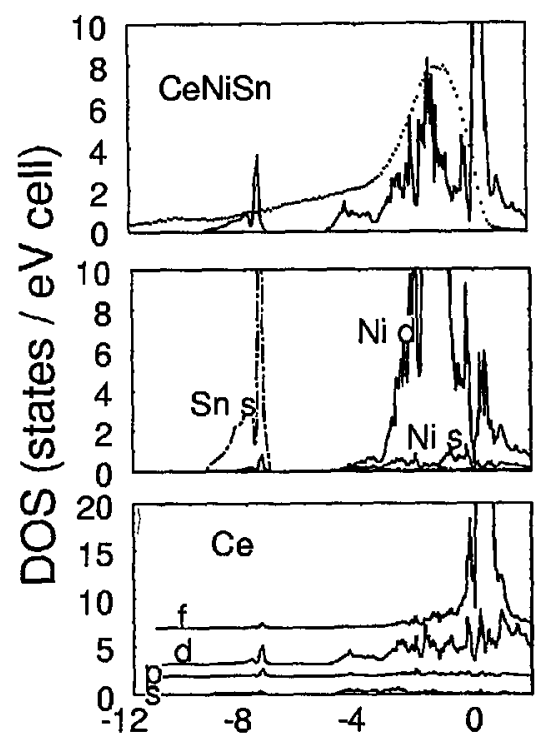

Fig. 2. Comparison of the total DOS and the measured XPS valence bands corrected by the background (dotted line) for CeNiSn. The partial DOS are plotted below.

and the Ce $4 f$-band states hybridization with the help of band-structure calculations. In Fig. 3 we compare the total DOS and the measured $h \nu=21.2$ and $40.8 \mathrm{eV}$ UPS spectra with the resolution near $\epsilon_{\mathrm{F}}$ of about two orders better than that of the XPS VB spectra. The V-shaped gap is strongly dependent on the $f$-conduction electron hybridization $[11,13]$. In band-structure calculations, the Ce $4 f$ electrons are treated as band electrons. In reality, they are more localized, and to treat them correctly, band theory would have to include strong local correlations. One way to 

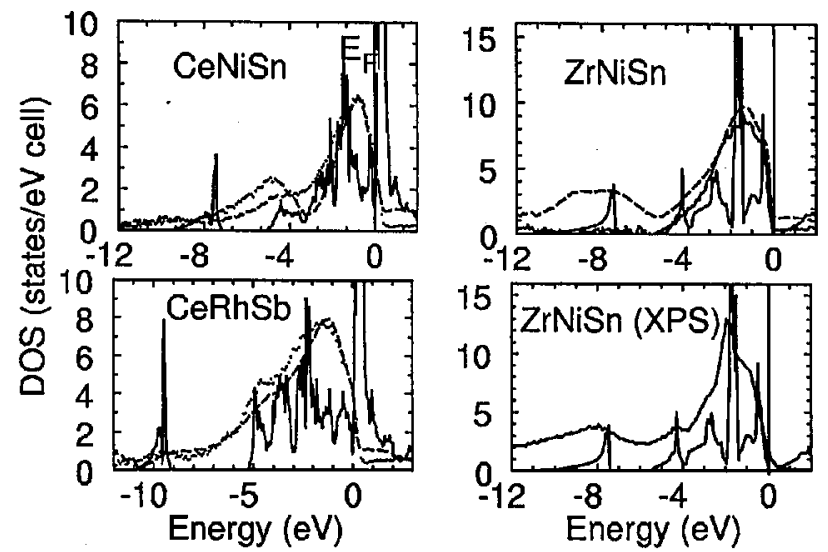

Fig. 3. Comparison of the total DOS (solid line) and the measured $h \nu=21.2$ (points) and $40.8 \mathrm{eV}$ (dashed line) UPS spectra.

explore this issue would be to carry out the calculations treating the Ce $4 f$ as a frozen core state, which does not hybridize with the conduction electrons. These correlations could cause the numerical changes in the band (e.g. the shift in the gap energy, the change of DOS at $\epsilon_{\mathrm{F}}$ ). Our calculations treating the Ce $4 f$ as a frozen core state have never given the gap [13]. We conclude that local correlations can have a destructive influence on the gap. This is a competitive effect to $f-d$ hybridization near $\epsilon_{\mathrm{F}}$, which can alter the DOS at the gap energy and form the pseudogap.

\subsection{Effect of alloying on the electronic structure}

The LMTO calculations show the destructive influence of the substituted element either in the $\mathrm{Ni}(\mathrm{Rh})$ or Ce atomic positions on the gap formation at $\epsilon_{\mathrm{F}}[19,20,11,13]$. Taking into consideration the boundary components of $\mathrm{CeNi}_{1-x} \mathrm{M}_{x} \mathrm{Sn}$ or $\mathrm{CeRh}_{1-x} \mathrm{M}_{x} \mathrm{Sn}$ ( $\mathrm{M}$ is $d$-metal), most of CeMSn or CeMSb in contrast to $\mathrm{CeNiSn}$ and $\mathrm{CeRhSb}$ are antiferromagnets at $T=0$. We suggest that the gap formation in Kondo insulators depends on the hybridization strength, but first of all on the energy of the $d$-subbands location in respect to $\epsilon_{\mathrm{F}}$. The alloying shifts the hybridization process to a higher energy in the bands.

The substitution of Ce by La decreases the number of $f$ electrons and the Fermi level continuously moves towards lower binding energies. The pseudo-V-shape gap is visible in the bands for all concentrations of La, and shifts with increasing $\mathrm{La}$ to higher energies in the nonoccupied part above $\epsilon_{\mathrm{F}}$. In Fig. 4 the calculated bands near $\epsilon_{\mathrm{F}}$ are presented for $\mathrm{Ce}_{1-x} \mathrm{La}_{x} \mathrm{NiSn}$ and for $\mathrm{Ce}_{1-x} \mathrm{La}_{x} \mathrm{RhSb}$. The alloying decreases valence of $\mathrm{Ce}$, which in the coherent ground state is always bigger than 3 . We argued in Refs. $[11,12]$ that the occupation number of Ce $f$ orbital, when it is smaller than 1 , correlates with formation of the gap. For $\mathrm{Ce}_{1-x} \mathrm{La}_{x} \mathrm{NiSn}$ compounds the occupation number $n_{f}$ is close to 1 , whereas for CeRhSb alloys $n_{f}<1$, even for $x=0.75$. In this case the calculations show the 
(a)

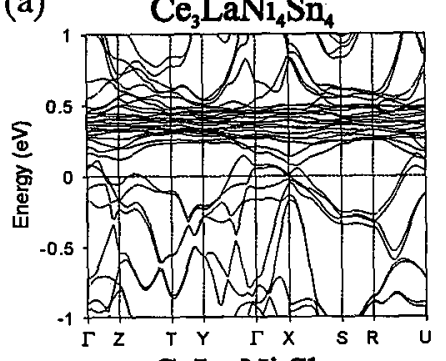

$\mathrm{CeLa}_{3} \mathrm{Ni}_{4} \mathrm{Sb}_{4}$

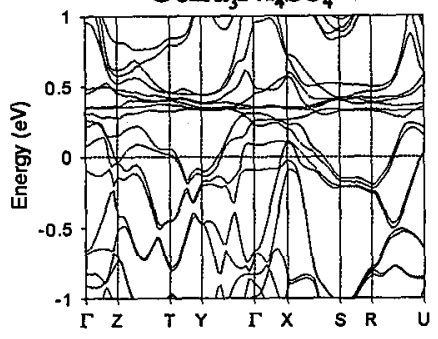

(b)

(b) $\quad \mathrm{Ce}_{3} \mathrm{LaRh}_{4} \mathrm{Sb}_{4}$
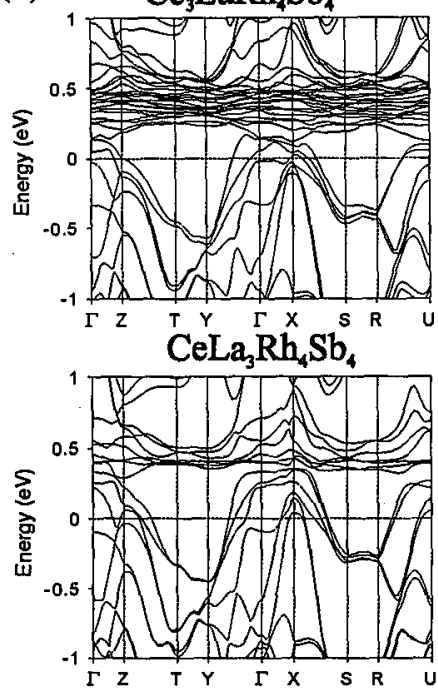

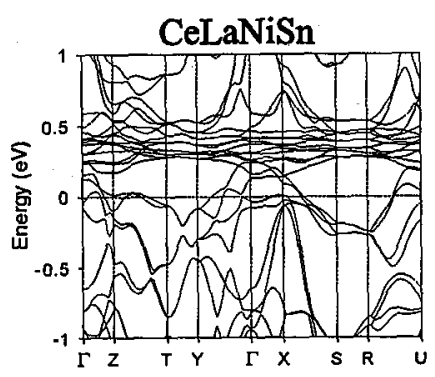

LaNiSn
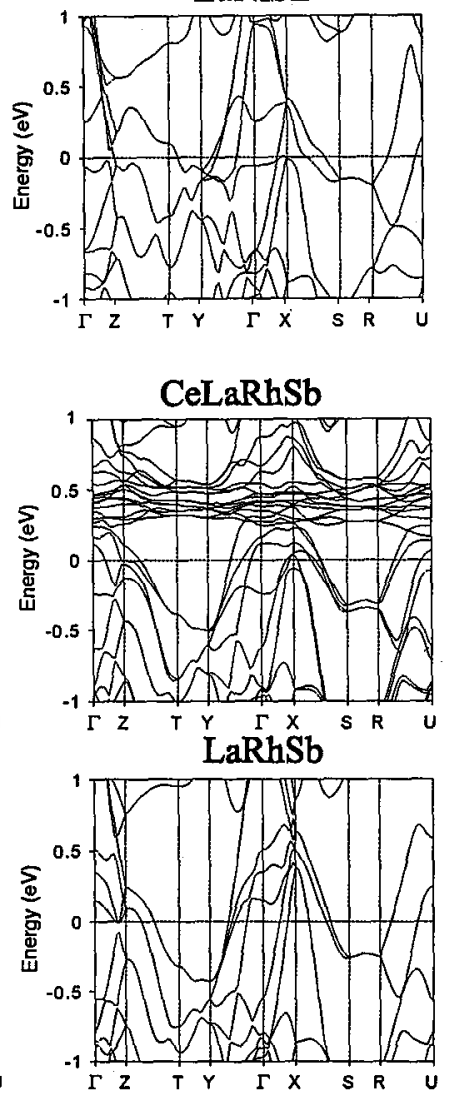

Fig. 4. TB LMTO band structure of $\mathrm{Ce}_{1-x} \mathrm{La}_{x} \mathrm{NiSn}$ (a) and $\mathrm{Ce}_{1-x} \mathrm{La}_{x} \mathrm{RhSb}(\mathrm{b})$ along various symmetry directions in the Brillouine zone (from Ref. [11]).

indirect gap above $\epsilon_{\mathrm{F}}$. A neutral charge substitution of Ce by a nonmagnetic impurity leads to the "Kondo hole" in the $f$ states. As it was shown in Ref. [31] a finite concentration of Kondo holes generates an impurity band in the gap that progressively smears the hybridization gap and also could have magnetic properties. The Ce $3 d$ XPS spectra indicated the mixed valence Ce ions in CeNiSn and $\mathrm{CeRhSb}$ and trivalent $\mathrm{Ce}$, when $\mathrm{Ce}$ is substituted by $\mathrm{La}$ in $\mathrm{Ce}_{1-x} \mathrm{La}_{x} \mathrm{NiSn}$. The $\mathrm{Ce}$ 
$3 d$ XPS spectra suggest the presence of the Kondo-hole contribution only in the bands of $\mathrm{Ce}_{1-x} \mathrm{La}_{x} \mathrm{RhSb}$ alloys, however, $\mathrm{Ce}_{1-x} \mathrm{La}_{x} \mathrm{NiSn}$ seem to be questionable Kondo-hole system in Ref. [31].

\section{3. $C e$ 3d XPS spectra}

Figure 5 shows the Ce $3 d$ XPS spectra of $\mathrm{Ce}_{1-x} \mathrm{La}_{x} \mathrm{RhSb}$ alloys. Three final-state contributions $f^{0}, f^{1}$, and $f^{2}$ are observed, which exhibit a spin-orbit splitting $\Delta_{\text {So }}$. The appearance of the $f^{0}$ components is clear evidence of the mixed valence. Based on the Gunnarsson-Schönhammer theoretical model [32, 33], the

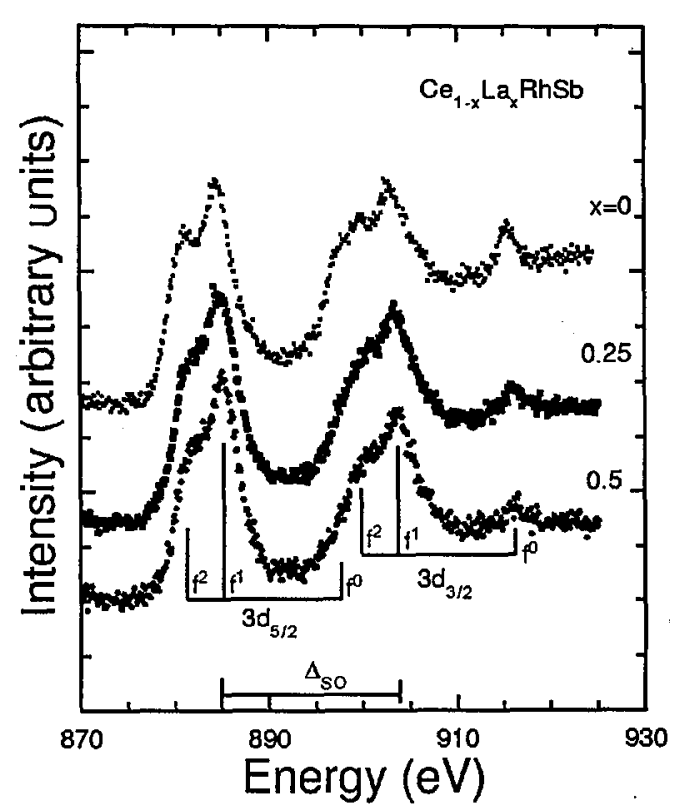

Fig. 5. Ce $3 d$ XPS spectra obtained for several $\mathrm{Ce}_{1-x} \mathrm{La}_{x} \mathrm{RhSb}$ alloys. The $3 d^{9} 4 f^{1}$, $3 d^{9} 4 f^{0}$, and $3 d^{9} 4 f^{2}$ components are separated on the basis of the Doniach-Sunjić theory.

intensity ratio $I\left(f^{0}\right) / I\left(f^{0}\right)+I\left(f^{1}\right)+I\left(f^{2}\right)$, which should be directly related to the $f$-occupation probability in the final states, indicates an $f$-occupation number $n_{f}^{\mathrm{XPS}}$. The $f^{2}$ components located at the low-binding-energy side of the $f^{1}$ components in the Ce XPS spectrum are attributed within the Gunnarsson-Schönhammer model to the hybridization between the $f$ states and the conduction band. The hybridization energy $\Delta=\Pi V^{2} \rho_{\max }$ describes the hybridization part of the Anderson impurity Hamiltonian, where $\rho_{\max }$ is the maximum in DOS and $V$ is the hybridization matrix element. Since the intensity ratio $r=I\left(f^{2}\right) /\left[I\left(f^{1}\right)+I\left(f^{2}\right)\right]$ has been calculated in Ref. [33] as a function of $\Delta$, it is possible to estimate the hybridization width $\Delta$. The separation of the peaks in the Ce $3 d$ XPS spectra, which overlap, was made on the basis of the Doniach-Sunjic theory [34]. The hy- 
bridization width $\Delta$ is about $150 \mathrm{meV}$ for CeNiSn and for CeRhSb with accuracy of $50 \mathrm{meV}$.

\subsection{Magnetic ground state}

The magnetic susceptibility of CeNiSn and CeRhSb is typical for a cerium valence-fluctuation compounds having a nonmagnetic ground state. In Fig. 6 we present as an exapmle $\chi$ of CeRhSb as a function of $T$. Below $50 \mathrm{~K} \chi$ shows an increase which corresponds to an incomplete solution of Ce in the alloy $[9,19,13]$. The ionic two-interconfigurational fluctuation model proposed by Sales and Wohlleben [35] can determine how much of the susceptibility reported in Fig. 6 is intrinsic. The solid lines represent the $\chi(T)=\chi_{0}+n C / T+N \mu^{2} n_{f}^{\chi} /\left[3 k_{\mathrm{B}}\left(T+T_{s f}\right)\right]$

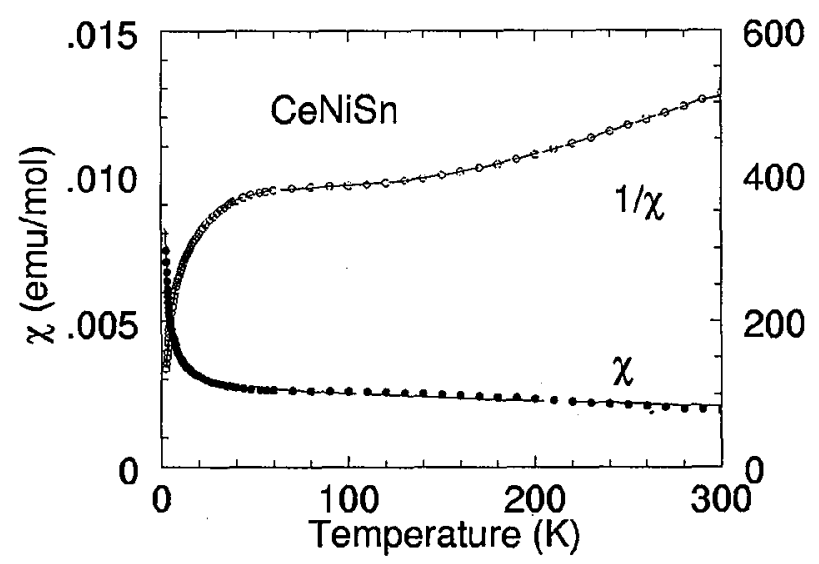

Fig. 6. Magnetic susceptibility $\chi$ in emu/mol and invers susceptibility $\chi^{-1}$ in $\left(\mathrm{cm}^{3} / \mathrm{mol}\right)^{-1}$. The applied magnetic field is $10 \mathrm{kOe}$. The solid line is a fit based on the ICF model with the parameters given in Table.

[and $\chi^{-1}(T)$ ] fits based on this model with parameters given in Table. A CurieWeiss term $n C / T$ explains the observed upturn in susceptibility caused by a fraction $n$ of paramagnetic Ce impurities. The valence fluctuation $n_{f}^{\chi}$ is computed based on a Boltzmann statistic for an ionic two-level system $\left(4 f^{1}, 4 f^{0}\right) . T_{s f}$ closed to $\Theta$ is interpreted in this model as the inverse of the valence fluctuation lifetime. The alloying $(x<1)$ does not change the nonmagnetic ground state.

\subsection{Comparison of the electronic structure of some ternary $d$ and $f$ electron intermetallics}

It is a well-known fact that for almost each Kondo insulator there is an isostructural semiconductor in which Ce (or mixed valence $f$-element) is replaced by a tetravalent non- $f$-element. ZrNiSn or TiNiSn are good examples of the isostructural semiconductors for CeNiSn or for CeRhSb. In Ref. [12] we have argued that the lowering of the valence of $\mathrm{Zr}$ or $\mathrm{Ti}$ in the Ce-dopped samples closes the gap at $\epsilon_{\mathrm{F}}$. We believe that the reasons leading to the gap in the semi-Heusler 
TABLE

The magnetic properties and the results of TB LMTO band structure calculations. The values of $\chi_{0}, n, T_{s f}$ and the excitation energy $E_{x} / k_{\mathrm{B}}$ are defined by fitting $\chi(T)$ to the experiment. Curie-Weiss temperature $\Theta$ is from the linear $\chi^{-1}$ relation vs. $T$.

\begin{tabular}{|c|c|c|c|}
\hline & $\mathrm{CeRhSb}$ & $\mathrm{CeRh}_{0.9} \mathrm{Co}_{0.1} \mathrm{Sb}$ & $\mathrm{CeRh}_{0.9} \mathrm{Pd}_{0.1} \mathrm{Sb}$ \\
\hline$\chi_{0}[\mathrm{emu} / \mathrm{mol}]$ & $7.4 \times 10^{-1}$ & $1.6 \times 10^{-2}$ & $4.1 \times 10^{-4}$ \\
\hline$n$ & 0.016 & 0.051 & 0.053 \\
\hline$\Theta[\mathrm{K}]$ & 290 & & 160 \\
\hline$T_{s f}$ & 250 & 143 & 124 \\
\hline$E_{x}[\mathrm{~K}]$ & 412 & 0 & 194 \\
\hline$n_{f}^{\chi}$ & 0.71 & 0.86 & 0.79 \\
\hline$n_{f}^{\mathrm{XPS}}$ & 0.86 & 0.89 & 0.86 \\
\hline$\Delta[\mathrm{meV}]$ & 140 & 170 & 150 \\
\hline \multirow{3}{*}{$\begin{array}{l}\text { DOS at } \epsilon_{F} \\
{[\text { states } /(\mathrm{eV} \text { at })]}\end{array}$} & 0.37 & 0.37 & 1.07 \\
\hline & & & \\
\hline & $\mathrm{CeRhSb}_{0.9} \mathrm{Sn}_{0.1}$ & CeNiSn & $\mathrm{CeNi}_{0.9} \mathrm{Cu}_{0.1} \mathrm{Sn}$ \\
\hline$\chi_{0}[\mathrm{emu} / \mathrm{mol}]$ & $3.1 \times 10^{-4}$ & $8 \times 10^{-4}$ & $3.9 \times 10^{-4}$ \\
\hline$n$ & 0.093 & 0.06 & 0.05 \\
\hline$\Theta[K]$ & 180 & 93 & 133 \\
\hline$T_{s f}$ & 132 & 120 & 76 \\
\hline$E_{x}[\mathrm{~K}]$ & 316 & 144 & 0 \\
\hline$n_{f}^{\chi}$ & 0.74 & 0.81 & 0.94 \\
\hline$n_{f}^{\mathrm{XPS}}$ & $<1$ & 0.95 & $<1$ \\
\hline$\Delta[\mathrm{meV}]$ & 160 & 150 & 150 \\
\hline $\begin{array}{l}\text { DOS at } \epsilon_{\mathrm{F}} \\
{[\text { states } /(\mathrm{eV} \text { at })]}\end{array}$ & 0.82 & 0.07 & 0.74 \\
\hline
\end{tabular}

alloys of the ZrNiSn-type and in the Ce-Kondo insulators seem to be similar. Very similar band properties as: strong hybridization, the gap formation at $\epsilon_{\mathrm{F}}$ and the second gap inside the valence bands, and the very similar VB XPS spectra allowed us to classify both alloys: $\mathrm{ZrNiSn}$ and CeNiSn to the common group of the intermetallics with the hybridization gap $[36,19]$. Moreover, the gap of the order of $400 \mathrm{meV}$ in $\mathrm{ZrNiSn}$ or TiNiSn is distroyed if $\mathrm{Ni}$ is replaced by another transition element, e.g. by Co. The volume thermal expansion coefficient of ZrNiSn and of TiNiSn shows two anomalous changes: one due to the change of the substitutional disorder between the $\mathrm{Zr}$ or $\mathrm{Ti}$ and $\mathrm{Sn}$ sublattice, the second is observed at $T<30 \mathrm{~K}$ and correlates with the resistivity abnormal increase in this temperature range. This abnormal change in $\rho(T)$ was attributed in Ref. [37] to the Mott-type behavior with $\rho=\rho_{0} \exp \left(T_{0} / T\right)^{1 / 4}$. For both semi-Heusler alloys the lattice parameters 
have a linear scaling with $\chi T$ (in Ref. [38]). The anomalous linear scaling is also observed for CeNiSn and for $\mathrm{CeRhSb}$ [38]. Several experiments, carried out independently, have indicated the same change of the resistivity below $T=40 \mathrm{~K}$ either for $\mathrm{CeRhSb}$ or for CeNiSn $[8,39,40]$. The coefficient of the volume expansion of $\mathrm{CeRhSb}$ has also shown a distinct contribution, which coincided with a change of resistivity [41]. The abnormal change of the volume expansion coefficient $\alpha$ seems to be analogous to that observed below $T=40 \mathrm{~K}$ in $\mathrm{ZrNiSn}$, therefore, we suggest to attribute the same reasons to explain the volume and resistivity anomalies at $T<40 \mathrm{~K}$ in the $\mathrm{ZrNiSn-type} \mathrm{semi-Heusler} \mathrm{alloys} \mathrm{and} \mathrm{in} \mathrm{CeNiSn-type} \mathrm{Kondo}$ insulators. If the Mott behavior indeed would be addressed to the abnormal low $T$ resistivity increase in $\mathrm{ZrNiSn}$ and TiNiSn, it also should explain the ground state of the Kondo insulator-type alloy. We know the Kondo insulator $\left(\mathrm{SmB}_{6}\right)$ as an example of striking parallel to Mott-Hubbard insulator such as $\mathrm{V}_{2} \mathrm{O}_{3}$ [42]. Spałek et al. [43] have presented the diagram on the $T-U / W$ plane of possible metal-insulator transitions. They discussed the possible transition from the paramagnetic metal to the paramagnetic insulator. However, the ground state always ought to be antiferromagnetic. We know several experiments, which only indicated the low temperature antiferromagnetic correlations in CeNiSn and CeRhSb, however, the antiferromagnetic ground state has not been observed yet. Therefore, in Ref. [38] we have suggested the concurrence of the Mott-type behavior and the Kondo hybridization in Ce-Kondo insulators.

\section{Conclussions}

The calculations of the electronic structure of the ZrNiSn-type alloys with tetravalent $\mathrm{Zr}$ and of CeNiSn-type Kondo insulators showed a similar energy distribution of DOS. The semiconducting gap was calculated for ZrNiSn, but for $\mathrm{CeNiSn}$ and $\mathrm{CeRhSb}$ there is a narrow pseudogap at the Fermi level. Very similar band properties as: strong hybridization, the gap at $\epsilon_{\mathrm{F}}$ and the second in the middle of the valence band, allowed us to classify both alloys: $\mathrm{ZrNiSn}$ and CeNiSn to the common group of the intermetallics with the hybridization gap. The gap is strongly reduced with alloying, the Ce $3 d$ XPS spectra suggest the presence of the Kondo-hole contribution in the bands of $\mathrm{Ce}_{1-x} \mathrm{La}_{x} \mathrm{RhSb}$.

\section{Acknowledgments}

We are most grateful to Dr Manfred Neumann (Fachbereich Physik, Universität Osnabrück) for discussions.

\section{References}

[1] A. Jayaraman, P. Denier, L.D. Longinotti, Phys. Rev. B 11, 2783 (1975).

[2] J.W. Allen, R.M. Martin, B. Batlogg, P. Wachter, J. Appl. Phys. 49, 2078 (1978).

[3] P. Haen, F. Lapierre, J.M. Mignot, R. Tournier, F. Holtzberg, Phys. Rev. Lett. 43, 304 (1979).

[4] M. Kasaya, F. Iga, M. Takigawa, T. Kasuya, J. Magn. Magn. Mater. 47-48, 429 (1985). 
[5] G.P. Meisner, M.S. Torikachvili, K.N. Yang, M.B. Maple, R.P. Guertin, J. Appl. Phys. 57, 3037 (1985).

[6] T. Palstra, J. Magn. Magn. Mater. 67, 331 (1987).

[7] S. Föex, J. Phys. Radium 9, 37 (1938); V. Jaccarino, G.K. Wertheim, J.H. Wernick, L.R. Walker, S. Arajs, Phys. Rev. 160, 476 (1967); Z. Schlesinger, Z. Fisk, H.T. Zhang, M.B. Maple, J.F. DiTusa, G. Aeppli, Phys. Rev. Lett. 71,1748 (1993).

[8] T. Takabatake, Y. Nakazawa, M. Ishikawa, Jpn. J. Appl. Phys, 26 Suppl., 547 (1987), T. Takabatake, F. Teshima, H. Fuji, S. Nishigori, T. Suzuki, T. Fujita, Y. Yamaguchi, J. Sakurai, D. Jaccard, Phys. Rev. B 41, 9607 (1990).

[9] S.K. Malik, D.T. Androja, Phys. Rev. B 43, 6277 (1991).

[10] M.F. Hundley, P.C. Canfield, J.D. Thompson, Z. Fisk, J.M. Lawrence, Phys. Rev. $B$ 42, 6842 (1990).

[11] A. Ślebarski, A. Jezierski, S. Mähl, M. Neumann, G. Borstel, Phys. Rev. B 58, 4367 (1998).

[12] A. Ślebarski, A. Jezierski, A. Zygmunt, S. Mähl, M. Neumann, Phys. Rev. B 57, 9544 (1998).

[13] A. Ślebarski, A. Jezierski, A. Zygmunt, S. Mähl, M. Neumann, Phys. Rev. B 58, 13498 (1998).

[14] Z. Fisk, J.L. Sarro, J.D. Thompson, D. Mandrus, M.H. Hundley, A. Miglori, B. Bucher, Z. Schlesinger, G. Aeppli, E. Bucher, J.F. DiTusa, C.S. Oglesby, H.R. Ott, P.C. Confield, S.E. Brown, Physica B 206/207, 798 (1995).

[15] Z. Fisk, P.C. Confield, J.D. Thompson, M.F. Hundley, J. Alloys Comp. 181, 369 (1992).

[16] R.M. Martin, Phys. Rev. Lett. 48, 362 (1982).

[17] F.G. Aliev, V.V. Moshchalkov, M.K. Zalyalutdinov, G.I. Pak, R.V. Skolozdra, P.A. Alekseev, V.N. Lazukov, I.P. Sadikov, Physica B 163, 3581 (1990).

[18] T. Takabatake, G. Nakamoto, H. Tanaka, H. Fujii, S. Nishigori, H. Goshima, T. Suzuki, T. Fujita, I. Oguro, T. Hiraoka, S.K. Malik, Physica B 199/200, 457 (1994).

[19] A. Ślebarski, A. Jezierski, A. Zygmunt, S. Mähl, M. Neumann, G. Borstel, Phys. Rev. B 54, 13551 (1996).

[20] A. Ślebarski, A. Jezierski, S. Mähl, M. Neumann, G. Borstel, Phys. Rev. B 56, 7245 (1997).

[21] F.G. Aliev, H.B. Brandt, V.V. Moshchalkov, M.K. Zalyalutdinov, R.V. Skolozdra, O.E. Koretskaya, G.I. Pak, Pis'ma Zh. Eksp. Teor. Fiz. 48, 536 (1988); Y. Uwatoko, T. Ishii, G. Oomi, H. Takahasi, N. Mori, J.D. Thompson, J.L. Shero, D. Madru, Z. Fisk, J. Phys. Soc. Jpn. 65, 27 (1996).

[22] O.K. Andersen, O. Jepsen, M. Sob, in: Electronic Structure and its Applications, Ed. M. Yussouff, Springer, Berlin 1987, p. 2.

[23] U. von Barth, L. Hedin, J. Phys. C 5, 1629 (1972).

[24] C.D. Hu, D.C. Langreth, Phys. Scr. 32, 391 (1985).

[25] T. Takanabake, Y. Nakazawa, M. Ishikawa, T. Sakakibara, K. Koga, I. Oguro, J. Magn. Magn. Mater. 76/77, 87 (1988).

[26] S. Nishigori, H. Goshima, T. Suzuki, T. Fujita, G. Nakamoto, T. Takabatake, H. Fujii, J. Sakurai, Physica B 186/188, 406 (1993). 
[27] M. Kurisu, T. Takabatake, H. Fujiwara, Solid State Commun. 68, 595 (1988).

[28] Y. Uwatoko, T. Ishii, G. Oomi, H. Takahasi, N. Mori, J.D. Thompson, J.L. Shero, D. Madru, Z. Fisk, J. Phys. Soc. Jpn. 65, 27 (1996).

[29] T. Ekino, T. Takabatake, H. Fujii, Physica B 230/232, 635 (1997).

[30] K. Nakamura, Y. Kitaoka, K. Asayama, T. Takabatake, G. Nakamoto, H. Tanaka, H. Fujii, Phys. Rev. B 53, 6385 (1996); K. Nakamura, Y. Kitaoka, K. Asayama, T. Takabatake, G. Nakamoto, H. Fujii, Phys. Rev. B 54, 6062 (1996).

[31] P. Schlottmann, Phys. Rev. B 54, 12324 (1996).

[32] O. Gunnarsson, K. Schönhammer, Phys. Rev. B 28, 4315 (1983).

[33] J.C. Fuggle, F.U. Hillebrecht, Z. Zołnierek, R. Lässer, Ch. Freiburg, O. Gunnarsson, K. Schönhammer, Phys. Rev. B 27, 7330 (1983).

[34] S. Doniach, M. Sunjić, J. Phys. C 3, 286 (1970).

[35] B.C. Sales, D. Wohlleben, Phys. Rev. Lett. 35, 1240 (1975).

[36] A. Ślebarski, A. Jezierski, S. Lütkehoff, M. Neumann, Phys. Rev. B 57, 6408 (1998).

[37] F.G. Aliev, H.B. Brandt, V.V. Kozyrkov, V.V. Moshchalkov, R.V. Skolozdra, Yu.V. Stadnyk, V.V. Pecharskii, Pis'ma Zh. Eksp. Teor. Fiz. 45, 535 (1987).

[38] A. Ślebarski, M. Orzechowska, A. Wrona, J. Szade, A. Jezierski, in press.

[39] Y. Uwatoko, T. Ishii, G. Oomi, H. Takahasi, N. Mori, J.D. Thompson, J.L. Shero, D. Madru, Z. Fisk, J. Phys. Soc. Jpn. 65, 27 (1996).

[40] S. Malik, L. Menon, V.K. Pecharsky, K.A. Gschneidner Jr., Phys. Rev. B 55, 11471 (1997).

[41] A.J. Nolten, A. de Visser, F.E. Kayzel, J.J.M. Franse, H. Tanaka, T. Takabatake, Physica B 206/207, 825 (1995).

[42] J.F. Cooley, M.C. Aronson, Z. Fisk, P.C. Confield, Phys. Rev. Lett. 74, 1629 (1995).

[43] J. Spałek, A. Datta, J.M. Honig, Phys. Rev. Lett. 59, 728 (1987); S.A. Carter, J. Yang, T.F. Rosenbaum, J. Spałek, J.M. Honig, Phys. Rev. B 43, 607 (1991). 